\title{
Derecho administrativo en el marco de la comunidad andina: la ampliación de la legitimidad activa para solicitar interpretaciones prejudiciales $\left.{ }^{*}\right)$
}

\author{
Administrative law in the framework of the andean community: the extension \\ of the locus standi to request preliminary rulings
}

\author{
Luis José Diez Canseco Núñez ${ }^{(*)}$ \\ Pontificia Universidad Católica del Perú (Lima, Perú) \\ María Ángela Sasaki Otani $i^{(* *)}$ \\ Pontificia Universidad Católica del Perú (Lima, Perú)
}

\begin{abstract}
Resumen: Uno de los temas de mayor trascendencia en el Derecho Administrativo comunitario andino está referido a la ampliación de la legitimidad activa para solicitar interpretaciones prejudiciales al Tribunal de Justicia de la Comunidad Andina (TJCA). De hecho, el TJCA ha interpretado de manera amplia y generosa el concepto de "juez nacional", incluyendo a los órganos administrativos de los Estados miembros, así como a los árbitros. De tal manera que, el problema de investigación gira en torno a qué se entiende por "juez nacional" a la luz del Derecho comunitario andino y el objeto y fin del mecanismo de la interpretación prejudicial. La hipótesis principal consiste en que, tanto los órganos administrativos como los árbitros son, en los hechos, "jueces comunitarios" por colaborar en la interpretación y la aplicación uniforme del Derecho comunitario. En ese sentido, en el presente trabajo se analizará la interpretación prejudicial como uno de los más importantes vehículos para garantizar el Estado de Derecho en materia de Propiedad Intelectual en el marco de la Comunidad Andina (CAN), y se hará un breve análisis comparativo entre la consulta prejudicial del Tribunal de Justicia de la Unión Europea (TJUE) y la del TJCA. Por último, se brindarán algunos apuntes sobre cómo se deben enfrentar los cambios jurisprudenciales del TJCA.
\end{abstract}

Palabras clave: Derecho Administrativo - Tribunal de Justicia de la Comunidad Andina - Interpretación prejudicial - Legitimidad activa - Régimen común sobre propiedad Intelectual

$\left(^{*}\right) \quad$ Nota del Editor: Este artículo fue recibido el 5 de marzo de 2021 y su publicación fue aprobada el 4 de mayo de 2021.

${ }^{(* *}$ Abogado por la Pontificia Universidad Católica del Perú (PUCP) y Magíster en Derecho Comparado por la George Washington University Law School. Decano de la Facultad de Derecho de la Universidad Tecnológica del Perú (UTP) y Profesor de la Facultad de Derecho de la PUCP. Socio Fundador del estudio de abogados Diez Canseco, Competencia y Propiedad Intelectual. Ha sido Magistrado Principal por la República del Perú y Presidente del Tribunal de Justicia de la Comunidad Andina (TJCA), Vocal de la Sala de Competencia del Tribunal del Instituto Nacional de Defensa de la Competencia y de la Protección de la Propiedad Intelectual del Perú (INDECOPI), funcionario de la Organización Mundial de la Propiedad Intelectual (OMPI) y de la Secretaría General de la Comunidad Andina. ORCID: https://orcid.org/0000-0002-2350-4645. Correo electrónico: Idiezcanseco@dclegal.pe.

$\left(^{* * *}\right.$ Abogada por la PUCP, Magister en Derecho Internacional Económico por la Universidad Andina Simón Bolívar (Ecuador) y Doctora en Derecho por la Universidad Complutense de Madrid. Ha sido Abogada Asesora de la Magistratura del Perú en el TJCA y Abogada de Presidencia de dicha corte internacional. También trabajó en el INDECOPI y en el Ministerio de Comercio Exterior y Turismo del Perú (MINCETUR) como negociadora internacional del capítulo de Propiedad Intelectual. Actualmente, es Asesora en el Ministerio de Relaciones Exteriores del Perú (MRE) y Profesora en la Facultad de Derecho de la PUCP. ORCID: https://orcid.org/0000-00021630-5242. Correo electrónico: sasaki.ma@pucp.edu.pe. 


\begin{abstract}
One of the most important issues in Andean Community administrative law concerns the extension of the locus standi to request preliminary rulings from the Court of Justice of the Andean Community (TJCA). In fact, the TJCA has interpreted the concept of "national judge" in a broad and generous manner, including the administrative bodies of the Member States, as well as arbitrators. Thus, the research problem revolves around what is understood by "national judge" in the light of Andean Community Law and the object and purpose of the mechanism of preliminary rulings. The main hypothesis is that administrative bodies and arbitrators are, in fact, "community judges" because they collaborate in the interpretation and uniform application of community law. In this sense, this paper will analyze the preliminary ruling interpretation as one of the most important vehicles to guarantee the rule of law in Intellectual Property matters within the framework of the Andean Community (CAN), and a brief comparative analysis will be made between the preliminary ruling of the Court of Justice of the European Union and that of the TJCA. Finally, some notes will be provided on how to deal with the changes in the case-law of the TJCA.
\end{abstract}

Keywords: Administrative Law - Court of Justice of the Andean Community - Preliminary Ruling - Locus Standi - Common Intellectual Property Regime

\section{Introducción: sobre el Tribunal de Justicia de la Comunidad Andina (TJCA)}

El 26 de mayo de 1969, Bolivia, Colombia, Chile, Ecuador y Perú suscribieron el Acuerdo de Cartagena, dando inicio al proceso de integración andino, el mismo que a la fecha tiene más de 50 años de existencia. A la fecha, los Países Miembros de la Comunidad Andina (CAN) son Bolivia, Colombia, Ecuador y Perú, sin perjuicio de que en un futuro puedan retornar Chile (fue País Miembro entre 1969 y 1976) y Venezuela (fue País Miembro entre 1973 y 2006) o puedan adherirse nuevos países.

En el marco del proceso de integración andino, los Países Miembros han transferido a esa organización internacional un conjunto de competencias soberanas; las mismas que caminan entre las normativas hasta las deliberantes. Estas características ponen de relieve la importancia asociativa de dicho esquema de integración. Al margen de los logros en materia económica y liberación del comercio, la CAN -junto con la UE- es uno de los procesos comunitarios más avanzados institucionalmente a nivel mundial. En efecto, en el ámbito de América Latina y El Caribe, la CAN ha conseguido lo que ningún otro bloque ha obtenido: que sus miembros estén dispuestos a ceder algunos aspectos de su soberanía en favor de la consecución de objetivos colectivos, comunes y superiores.

De la estructura jurídica comunitaria andina, uno de sus mayores logros se verifica mediante la creación del Tribunal de Justicia del Acuerdo de Cartagena el 28 de mayo de 1979, actualmente, Tribunal de Justicia de la Comunidad Andina (TJCA). Esta corte internacional se adscribe al modelo desarrollado en el Tribunal de Justicia de la Unión Europea (TJUE, antiguo Tribunal de Justicia de la Comunidad Europea - TJCE) y constituye el organismo más activo y de mayor productividad en el denominado Sistema Andino de Integración (SAI). En efecto, desde hace casi dos décadas es la tercera corte internacional con mayor actividad jurisdiccional dentro del panorama de cortes internacionales a nivel mundial, solo siendo menos activo que el Tribunal Europeo de Derechos Humanos (TEDH) y el Tribunal de Justicia de la UE (TJUE) respectivamente; pero siendo largamente más dinámico que la Corte Internacional de Justicia $(\mathrm{ClJ})$, las instituciones del sistema de solución de diferencias de la Organización Mundial de Comercio (OMC) y otras cortes internacionales (Alter \& Helfer, 2017).

Del mismo modo, sobre la actividad judicial de otras cortes internacionales de América Latina y El Caribe, se puede afirmar que la solución de controversias resulta más que preponderante en el ámbito del TJCA. Así, tiene la mayor carga procesal(1) en comparación con la Corte Interamericana de Derechos Humanos ${ }^{(2)}$, el Tribunal Permanente de Revisión (TPR) del MERCOSUR ${ }^{(3)}$,

(1) En cuanto al volumen de su actividad jurisdiccional, hasta febrero de 2021, el TJCA ha recibido un total histórico de 6441 procesos. Información oficial solicitada de manera directa a la Secretaría del TJCA en marzo de 2021.

(2) Hasta 2019, la Corte Interamericana de Derechos Humanos se ha pronunciado en 402 casos contenciosos, 643 medidas provisionales, 594 supervisiones de cumplimiento de sentencia y 26 opiniones consultivas. http://www.corteidh.or.cr/cf/Jurisprudencia2/busqueda_ casos contenciosos.cfm?lang=e

(3) A la fecha, en el seno del MERCOSUR se han dictado 25 decisiones judiciales: el Tribunal Permanente de Revisión (TPR) ha emitido 13 providencias, el Tribunal Ad Hoc del Protocolo de Olivos 2 providencias y el Tribunal Ad Hoc del Protocolo de Brasilia 10 providencias. http://www.tprmercosur.org/es/sol_contr_laudos.htm. 
la Corte Centroamericana de Justicia del Sistema de la Integración Centroamericana (SICA) ${ }^{(4)}$ y la Corte de Justicia de la Comunidad del Caribe (CARICOM) ${ }^{(5)}$. Del mismo modo, en lo que respecta al ámbito de responsabilidades, el TJCA es el más completo, puesto que se manifiesta a través de sentencias en los diversos procesos a su cargo, los cuales son similares a los del Tribunal de Justicia de la UE (TJUE).

Los poderes primigenios del TJCA se expresan a través de la resolución de las acciones de nulidad e incumplimiento, así como la interpretación prejudicial. En 1996 se incorporaron otros poderes, como la excepción de inaplicación, el recurso por omisión o inactividad, la función arbitral y la jurisdicción laboral (Protocolo Modificatorio del Tratado de Creación del Tribunal de Justicia de la Comunidad Andina, 1996).

En paralelo a su labor jurisdiccional, una de las principales responsabilidades del TJCA consiste en difundir el conocimiento del ordenamiento jurídico andino. Esta labor se ha intensificado exponencialmente desde el 2013 a través de infinidad de cursos, conferencias, seminarios, conversatorios, paneles y demás actividades académicas destinadas a los círculos judiciales, universitarios, profesionales, gremiales y empresariales, en los que se ha difundido el ordenamiento jurídico andino y la labor jurisdiccional del TJCA ${ }^{(6)}$. Ello ha repercutido en un incremento más que significativo en su carga procesal, principalmente en relación con las interpretaciones prejudiciales, que componen aproximadamente el $90 \%$ de su actividad jurisdiccional. La labor de difusión surgió como consecuencia de un cambio radical de perspectiva sobre qué se debía hacer en el seno del TJCA (Diez Canseco, 2019, p. 155 y ss.).

En efecto, la actividad resolutiva del TJCA se ha incrementado de forma constante y significativa: En 2014 se resolvieron 327 causas judiciales (19.7\% más que el 2013), en 2015 se resolvieron 479 causas judiciales (16.3\% más que el 2014), en 2016 se resolvieron 504 causas judiciales (5\% más que el 2015), en 2017 se resolvieron 556 causas judiciales (10\% más que el 2016), en 2018 se resolvieron 619 causas judiciales (11\% más que el 2017), y en 2019 se alcanzó la cifra histórica de 734 causas judiciales resueltas (19\% más que el 2018) (TJCA, 2020, p. 4). En el 2020, durante la pandemia del Covid-19, se resolvieron un total de 439 causas $(40 \%$ menos que en el 2019).

Así, desde 1984 -cuando entra en funciones el TJCA- hasta febrero de 2021, el TJCA ha recibido un total histórico de 6441 procesos y desde el 2013 hasta ahora se han visto 4324 casos.
Ello significa que en los últimos 8 años la carga procesal del TJCA ha aumentado en un sorprendente $67 \%$.

En particular, sobre la actividad jurisdiccional del TJCA se puede advertir que desde el 2014 se ha incrementado de manera exponencial: Si entre el 2008 y 2013 habían ingresado al año un promedio de 172 solicitudes, en el 2014 dicho número se duplicó e ingresaron 325 consultas. Esta tendencia permaneció durante el 2015, cuando se logró la cifra histórica de 691 consultas enviadas por los órganos jurisdiccionales, las entidades administrativas y los tribunales arbitrales de los cuatro Países Miembros: 397 de Colombia, 218 del Perú, 42 del Ecuador y 34 de Bolivia (TJCA, 2016, p. 7).

La justificación de la presente investigación radica en que, prácticamente, se ha cuadriplicado el número de solicitudes de interpretación prejudicial y ha aumentado la participación de Bolivia (país que había enviado únicamente dos solicitudes en el 2011 y seis en el 2014). En ese sentido, desde el 2015 se ha verificado un llamativo aumento de las solicitudes, que cabe atribuir a una importante labor de difusión del ordenamiento andino llevada a cabo por el TJCA (TJCA, 2016, pp. 7-8; pp. 65-66).

Al respecto, uno de los temas de mayor trascendencia en el Derecho Administrativo comunitario andino está referido, precisamente, a la ampliación de la legitimidad activa para solicitar interpretaciones prejudiciales al TJCA. De hecho, el TJCA ha interpretado de manera amplia y generosa el concepto de "juez nacional", incluyendo a los órganos administrativos de los Estados miembros, así como a los árbitros. De tal manera que, el problema de investigación gira en torno a la interrogante ¿qué se entiende por "juez nacional"?

Como metodología y técnica de investigación se realizará un breve paralelismo

(4) Hasta el 2017, la Corte Centroamericana de Justicia del Sistema de la Integración Centroamericana (SICA) habría emitido aproximadamente 170 decisiones judiciales. Véase sus memorias de labores: http://portal.ccj.org.ni/ccj/memorias/.

(5) Hasta el 2014, la Corte de Justicia de la Comunidad del Caribe (CARICOM) había pronunciado 76 sentencias. http://www. caribbeancourtofjustice.org/judgments-proceedings/original-jurisdiction- judgments.

(6) Entre el 2015 y el 2019 se dieron 127 eventos académicos y de difusión en los que participaron los Magistrados del TJCA. Información oficial solicitada de manera directa a la Secretaría del TJCA en junio de 2020. 
entre la consulta prejudicial del Tribunal de Justicia de la UE (TJUE) y la del TJCA (método comparativo). La razón de ser de esta aproximación metodológica consiste en que resulta necesario comparar el caso andino con el caso europeo, ya que no se podría hablar plenamente de la interpretación prejudicial andina, sin antes tomar como elemento referencial el sistema europeo, al ser la Unión Europea el proceso de integración más avanzado y nuestro único punto de referencia; mas ello, no debe implicar tomar tal modelo de manera ciega y mecánica, dejando de lado las particularidades y singularidades de la subregión andina.

Se recurrirá, además, a los principios de interpretación sistemática y teleológica de las normas, salvo que su aplicación se exceptúe por otros principios de interpretación del Derecho comunitario que, naturalmente, serán de aplicación preferente.

El objetivo del trabajo es vislumbrar una respuesta a la interrogante qué se entiende por "juez nacional" a la luz del Derecho comunitario andino y el objeto y fin del mecanismo de la interpretación prejudicial. La hipótesis principal consiste en que, tanto los órganos administrativos como los árbitros, cuentan con legitimidad activa para solicitar interpretaciones prejudiciales en la CAN, puesto que, en los hechos, son verdaderos "jueces comunitarios" al colaborar en la interpretación y la aplicación uniforme del Derecho comunitario.

A continuación, en el presente trabajo se analizará la interpretación prejudicial como uno de los más importantes vehículos para garantizar el Estado de Derecho en materia de Propiedad Intelectual en el marco de la CAN, y se hará un breve análisis comparativo entre la consulta prejudicial del TJUE y la del TJCA. Por último, se brindarán algunos apuntes sobre cómo se deben enfrentar los cambios jurisprudenciales del TJCA.

\subsection{La interpretación prejudicial como mecanismo garante de un Estado de Derecho de Propiedad Intelectual en la Comunidad Andina (CAN)}

Una de las tendencias modernas del Derecho administrativo se vincula con el reconocimiento de su existencia más allá de las fronteras de un Estado. De manera similar a la Unión Europea, el Derecho administrativo comunitario andino se nutre de la existencia de una función administrativa andina, una administración pública comunitaria, un sistema de fuentes andino y un contencioso administrativo comunitario (Santos, 2013, pp. 21-44).

Si bien la interpretación prejudicial constituye un mecanismo de colaboración judicial, en la práctica no se puede obviar su arista de mecanismo de control judicial de las actuaciones de los Países Miembros y de sus respectivas administraciones públicas. Como lo indica su nombre, tienen por objeto interpretar las normas que conforman el ordenamiento jurídico de la CAN con el fin de asegurar su aplicación uniforme en el territorio de los Países Miembros (Estatuto del TJCA - ETJCA, art. 121).
En ese sentido, el juez nacional no puede arrogarse el lugar del TJCA y comprometer la uniformidad en la interpretación y la aplicación de los tratados, además del derecho derivado de los órganos comunitarios.

De lo señalado hasta ahora se desprende que el objetivo principal de la cooperación judicial entre jueces nacionales y el TJCA consiste en evitar las posibles asimetrías interpretativas que se generarían si cada juez nacional de los cuatro Países Miembros decidiera hacer prevalecer su propia visión en relación con la manera como debe aplicarse el Derecho comunitario andino. En otras palabras, se trata de resguardar la igualdad entre los litigantes andinos si determinadas normas comunitarias se aplican de manera diferente.

\subsubsection{La interpretación prejudicial}

En el art. 121 del ETJCA se prescribe que: "Corresponde al Tribunal interpretar las normas que conforman el ordenamiento jurídico de la Comunidad Andina, con el fin de asegurar su aplicación uniforme en el territorio de los Países Miembros".

Sobre la relación entre el "juez nacional" y el TJCA la Corte Constitucional de la República de Colombia ha tenido ocasión de manifestar:

El sistema judicial comunitario, no se compone únicamente del Tribunal del Acuerdo de Cartagena, sino que conforma un concepto de mayor alcance, comprensivo tanto de las jurisdicciones de los Estados miembros como del Tribunal de Justicia. El tribunal no es el único que tiene jurisdicción comunitaria en cuanto a aplicaciones del derecho se refiere, por lo cual debe compartir sus funciones con las justicias nacionales sin afectar la autonomía judicial en los Estados miembros. (...) El papel del juez nacional en este sentido se convierte en pieza clave, puesto que por el camino de la interpretación prejudicial este se consolida como la instancia más inmediata que tiene el ciudadano para la aplicación del derecho de integración. Las relaciones interjurisdiccionales entre los tribunales comunitarios y las justicias nacionales se aplican básicamente en el ejercicio de la función de interpretación prejudicial que otorga a estas últimas el derecho de recurrir en consulta al tribunal en asuntos atinentes a la ley de la Comunidad, por una parte, y, por otra, 
la obligación de aplicar la interpretación efectuada por el Tribunal (Sentencia de la Corte Constitucional de Colombia, 25 de mayo de 1995, Expediente D-576).

La interpretación realizada por el TJCA tiene carácter obligatorio y vincula al consultante, en el sentido que este deberá brindar a la normativa comunitaria, dentro del proceso o procedimiento nacional, el significado y el alcance recogido y decidido por el Tribunal (ETJCA, art. 127).

La consulta puede ser facultativa u obligatoria. Será facultativa para los jueces nacionales que conozcan de un proceso en el que se controvierta alguna de las normas que conforman el ordenamiento jurídico de la CAN, pero que no son de única o última instancia ordinaria (ETJCA, art. 122). En cambio, si se trata de un proceso en el que la sentencia fuera de única o última instancia, que no fuere susceptible de recursos ordinarios en el Derecho interno y en el que deba aplicarse o se controvierta alguna de las normas andinas; el juez nacional deberá suspender el proceso y solicitar obligatoriamente la interpretación al Tribunal (ETJCA, art. 123).

Recibida la consulta, dentro del término de 30 días siguientes de su admisión por parte del TJCA, este dictará sentencia. En ella el TJCA deberá limitarse a precisar el contenido y el alcance de las normas andinas referidas al caso concreto. EI TJCA no interpretará el derecho nacional ni calificará los hechos materia del proceso o procedimiento nacional. Sin embargo, podrá referirse a estos cuando ello sea indispensable para efectos de emitir la interpretación solicitada (ETJCA, art. 126).

Cuando el juez nacional obligado a enviar la solicitud se abstenga de hacerlo o cuando, efectuada esta, se separe de la interpretación del TJCA, los Países Miembros y los particulares afectados podrán activar ante la SGCA un procedimiento administrativo denominado como acción de incumplimiento (ETJCA, art. 128; Cienfuegos, 2013). Sin embargo, la práctica ha demostrado que ello no suele suceder, puesto que mayormente no se cumple con esta obligación de fiscalización y control por parte de los Países Miembros y la SGCA.

Por otra parte, en cualquiera de los dos supuestos señalados anteriormente, los particulares podrán interponer una demanda de nulidad de sentencia en sede nacional por la contravención de una norma procesal de orden público, puesto que constituye una clara violación al debido proceso (Gálvez, 2001, pp. 140-143).

Sobre este punto, la jurisprudencia andina ha señalado que:

[e]l requisito de la solicitud de interpretación prejudicial, teniendo en cuenta la aplicación inmediata y el efecto directo de la normativa comunitaria andina, entra a formar parte de la normativa procesal nacional de manera inmediata y automática. Su incumplimiento acarrearía la nulidad de la sentencia y entraría a ser parte integrante de las causales de nulidad previstas en la normativa interna. Es importante recordar que la violación de las normas procesales es la base para alegar una violación al 'derecho al debido proceso'. De conformidad con las figuras procesales internas (...) recursos extraordinarios de revisión, casación (...) acciones constitucionales como la tutela, el amparo, la acción de protección, etc. De conformidad con las previsiones del Derecho interno, los afectados por este incumplimiento podrían solicitar la reparación del daño causado, utilizando, para el efecto, las figuras procesales nacionales (Proceso 248-IP-2013, pp. 13-14).

\subsubsection{Sobre un Estado de Derecho de Propiedad Intelectual en la CAN.}

Las interpretaciones prejudiciales señalan el derrotero y expresan los avances positivos hacia la consolidación del proceso de integración jurídica de la CAN, a diferencia de las acciones de incumplimiento y de nulidad, surgidas como consecuencia de las fallas o imponderables del proceso de integración. En efecto, el mayor número de sentencias de interpretación prejudicial pone de relieve que la normativa pertinente se aplica rigurosamente y es respetada. Del mismo modo, ratifica que el sector económico dentro de la que se encuentra es sumamente activo, además de que su normativa resulta de importancia. En cambio, las acciones de incumplimiento y de nulidad, tal como se ha indicado, suponen que no se está respetando el ordenamiento jurídico andino.

Asimismo, partiendo de la constatación de que las interpretaciones prejudiciales sirven para resolver problemas entre particulares, pone de relieve inequívocamente que se están protegiendo directamente los intereses individuales de los ciudadanos andinos, de los empresarios y de los consumidores, garantizando sus derechos e intereses y aportando beneficios tangibles. Sobre este particular también cabe señalar que en la medida que el sistema de integración se consolide y haya un mayor número de decisiones en otras materias, se tendrá como correlato un incremento aún mayor en el número de interpretaciones prejudiciales.

Sumado a la importante labor de difusión del Derecho comunitario llevada a cabo por el TJCA, el aumento exponencial de la carga procesal del TJCA se debe también en gran medida a la creación de la 
Octava Sala Especializada en lo Contencioso Administrativo de la Corte Superior de Justicia de Lima de la República del Perú (hoy Quinta Sala) y, particularmente debido a la ampliación de la legitimidad activa de las oficinas nacionales competentes para solicitar interpretación prejudicial facultativa. En efecto, se han recibido solicitudes de las entidades administrativas vinculadas con la propiedad intelectual de todos los Países Miembros; como es el caso del Servicio Nacional de Propiedad Intelectual del Estado Plurinacional de Bolivia (SENAPI) (Proceso 105IP-2014), de la Superintendencia de Industria y Comercio de Colombia (SIC)(Proceso 242-IP-2015), del Órgano Colegiado de Derechos Intelectuales del Servicio Nacional de Derechos Intelectuales del Ecuador (SENADI)(Proceso 737-IP-2018) y del Instituto Nacional de Defensa de la Competencia y de la Protección de la Propiedad Intelectual del Perú (INDECOPI)(Proceso 121-IP-2014 y Proceso 297IP-2014), tal como se verá más adelante.

Como lo demuestran las estadísticas, los conflictos que versan sobre la protección de la Propiedad Intelectual (PI) representan casi el $90 \%$ de las responsabilidades jurisdiccionales del TJCA (TJCA, 2016, pp. 71-75; TJCA, 2018, p. 10). Por ello, la jurisprudencia de dicho tribunal comunitario en materia de Propiedad Intelectual ha venido consolidando sistemáticamente la aplicación uniforme de esta clase de normativa comunitaria en todos los Países Miembros, además de que ha coadyuvado a establecer una isla de jurisdicción internacional eficaz y un Estado de Derecho de Propiedad Intelectual en la CAN (Alter et al, 2009, p. 2).

Cabe señalar que ha sido reconocido internacionalmente que los países andinos cuentan con un robusto sistema de $\mathrm{PI}$, reconocida en el art. 55 del Acuerdo de Cartagena y está expresado en sus dos grandes vertientes: los derechos de autor (Decisión 351) y la propiedad industrial (Decisiones 486, 632 y 689), además de un régimen común de protección a los derechos de obtentores de variedades vegetales (Decisiones 345 y 366) y un régimen común sobre acceso a los recursos genéticos (Decisiones 391, 423 y 448). Este último reconoce los derechos de las comunidades indígenas, afroamericanas y locales sobre sus conocimientos, innovaciones y prácticas tradicionales asociados con los recursos genéticos y sus productos derivados ${ }^{(7)}$. En cuanto a la propiedad industrial existe un denominado Régimen Común, el mismo que se expresa a través de la Decisión 486 de la Comisión de la CAN; la misma que fue adoptada en Lima el 14 de septiembre de 2000, entrando en vigor el 1 de diciembre de 2000 (Sasaki, 2014, pp. 67 y ss).

\section{Evolución jurisprudencial del TJCA en relación con la ampliación de la legitimidad activa para solicitar interpretaciones prejudiciales al TJCA}

El mecanismo de la interpretación prejudicial constituye una acción de colaboración y cooperación entre el juez nacional y el juez comunitario. La interpretación es solicitada por el "juez nacional" de un País Miembro que está conociendo un litigio y en el que, para resolverlo, debe aplicar el Derecho de la CAN. Lo importante del tema es que el TJCA ha interpretado de manera amplia y generosa el concepto de "juez nacional", al haber incluido, tal como se verá a continuación, a los órganos administrativos de los Países Miembros, así como a los árbitros, respectivamente.

\subsection{Los árbitros}

En un primer momento y hace más de una década, que en el marco del Proceso 3-Al2010, el TJCA determinó por primera vez la obligación de un tribunal arbitral de solicitar interpretación prejudicial de la siguiente manera:

Con ocasión de los procesos 14-IP-2007 y 130-IP-2007, este Tribunal comunitario amplió el concepto de juez nacional, a efectos de determinar quiénes podían solicitar una interpretación prejudicial. En este sentido, se incluyó dentro de este concepto a las entidades administrativas que cumplan funciones jurisdiccionales, como era el caso de la Superintendencia de Industria y Comercio de Colombia, Grupo de Trabajo de Competencia Desleal, que solicitó la interpretación prejudicial. Es decir, el Tribunal amplió el alcance del concepto de juez nacional a todas las entidades administrativas que cumplan funciones jurisdiccionales y que actúen como única o última instancia ordinaria. En este contexto, deriva necesario determinar si los árbitros ejercen funciones jurisdiccionales $y$, por tanto, se encuentran incluidos dentro del concepto anterior.

(7) Véase http://www.comunidadandina.org/Seccion.aspx?id=301\&tipo=TE\&title=propiedad-intelectual, consultado por última vez el 15 de julio. 
En efecto, el TJCA determinó que, si los árbitros tienen funciones jurisdiccionales y fungen como última o única instancia ordinaria, para los efectos del Derecho comunitario actúan como verdaderos jueces nacionales. Así, debido a esta interpretación amplia de la norma, se ha incluido a los árbitros dentro del concepto del "juez nacional", ya que ellos deciden en derecho. Por lo tanto, el TJCA estableció que los árbitros tienen la obligación de solicitar la interpretación prejudicial, de manera directa, sin la mediación de los órganos jurisdiccionales nacionales (Proceso 3-AI-2010).

Este criterio jurídico interpretativo ha sido recogido en el "Reglamento que regula aspectos vinculados con la solicitud y emisión de Interpretaciones Prejudiciales" aprobado por Acuerdo 08/2017, modificado por Acuerdo 04/2018 del TJCA, que al definir "órgano jurisdiccional" señaló que comprende a los árbitros.

Recientemente, mediante el Proceso 01-IP-2021, publicado el 6 de mayo de 2021 en la Gaceta Oficial del Acuerdo de Cartagena, el TJCA aclaró lo siguiente:

Lo que se debe evitar es que un árbitro o tribunal arbitral, al resolver una disputa aplicando el derecho andino, aplique criterios jurídicos interpretativos opuestos al de otro árbitro o tribunal arbitral, diferentes al aplicado por una autoridad administrativa o judicial, o distintos a los establecidos por el TJCA, por lo que resulta necesario que sea esta corte internacional, en ejercicio de sus competencias exclusivas y excluyentes, la que uniformice tales criterios jurídicos, lo que se efectúa, precisamente, a través de la interpretación prejudicial.

EI TJCA agregó que el hecho de que una controversia derive de una situación o relación jurídica cubierta por el derecho andino no significa, de antemano, que el árbitro o tribunal arbitral tenga que aplicar o que se controvierta dicho derecho en el momento de resolver la controversia. Hay escenarios en los cuales, si bien la cobertura jurídica la brinda el derecho andino, la controversia en sí misma incide sobre aspectos contractuales que no requieren que el árbitro o tribunal arbitral tenga que desarrollar un criterio jurídico interpretativo del derecho andino para resolverla. En efecto, si bien un asunto puede estar mediatamente relacionado con el derecho andino, lo cierto es que existen controversias que pueden ser resueltas sin necesidad de establecer, crear o aplicar un criterio jurídico interpretativo de la norma andina, mientras que otras disputas requieren, necesariamente para la resolución del caso, del establecimiento, creación o aplicación de un criterio jurídico interpretativo de la norma andina.

El Tribunal señaló que: "si para resolver la controversia que tiene en sus manos, el árbitro o tribunal arbitral no va a necesitar establecer, crear o aplicar un criterio jurídico interpretativo de la norma andina, no tiene la obligación de solicitar al TJCA interpretación prejudicial alguna. Si el árbitro o tribunal arbitral no va a establecer, crear o aplicar un criterio jurídico interpretativo de la norma andina, no hay forma en que se genere una aplicación diferenciada de la norma andina, por lo que en este caso no cabe aplicar el instituto de la interpretación prejudicial obligatoria".

En otras palabras, la mera alegación de una de las partes de una norma andina no genera la obligatoriedad de solicitar una interpretación prejudicial a cargo del TJCA. Para que sea exigible dicha solicitud, es imprescindible que el árbitro o tribunal arbitral tenga que aplicar o interpretar la norma andina para resolver la controversia, lo que significa la necesidad de establecer, crear o aplicar un criterio jurídico interpretativo de alguna disposición del ordenamiento jurídico comunitario andino para solucionar la disputa de que se trate.

EI TJCA cierra el Proceso 01-IP-2021 precisando que:

Lo expuesto en la presente interpretación prejudicial tiene el propósito de brindar una orientación más clara sobre lo establecido por el TJCA en la Sentencia recaída en el Proceso 03-Al-2010, oportunidad en la cual sustentó, con acierto, que los árbitros o tribunales arbitrales que, al resolver la controversia puesta a su conocimiento, van a aplicar el derecho andino, califican como "jueces nacionales" para efectos de la solicitud de interpretación prejudicial. (...) Resta señalar que los criterios jurídicos interpretativos establecidos en la presente sección se aplican "ex nunc", a partir de la publicación de la presente interpretación prejudicial en la Gaceta Oficial del Acuerdo de Cartagena. No tienen, por tanto, efecto retroactivo.

\subsection{Los órganos administrativos encargados del registro de la Propiedad Industrial}

En un segundo momento y a partir del Proceso 121-IP-2014 ${ }^{(8)}$, el TJCA también ha interpretado de manera amplia el concepto de "juez nacional"; incluyendo por primera vez a los órganos o entidades administrativas de los Países Miembros. Ello se detalla a continuación.

(8) Magistrado ponente: Luis José Diez Canseco Núñez. Interpretación prejudicial solicitada por la Dirección de Signos Distintivos (DSD) del Instituto Nacional de Defensa de la Competencia y de la Propiedad Intelectual del Perú (INDECOPI). 
En efecto, según el TJCA, "el concepto de 'juez nacional' constituye un concepto autónomo, propio del Derecho comunitario andino, que debe ser definido únicamente por el Tribunal de acuerdo con criterios propios" (Proceso 121-IP2014) y tomando en cuenta el objetivo mismo del mecanismo de la interpretación prejudicial.

Del mismo modo, el TJCA, en resguardo de su misión de asegurar la interpretación y la aplicación uniforme de las normas andinas en todos los Países Miembros, no se ha limitado, como lo hizo en el caso de los árbitros, a realizar una interpretación restrictiva y literal del art. 33 de su Tratado de Creación y de los arts. 122 y 123 de su Estatuto, sino que "ha ido ampliando el alcance del concepto de 'juez nacional' a la luz del objetivo final de la interpretación prejudicial; considerando que se trata de un término genérico y comprensivo de todas las autoridades que administran justicia por mandato legal" (Proceso 121-IP2014, p. 6).

Igualmente, en el marco de los procesos 14-IP-2007 y 130 IP-2007, el TJCA se pronunció sobre la legitimidad activa de la Superintendencia de Industria y Comercio de la República de Colombia (Grupo de Trabajo de Competencia Desleal) de la siguiente manera:

Se hace evidente que en la actualidad para clasificar la naturaleza de los actos ya no es suficiente el criterio orgánico y, en consecuencia, para analizar la naturaleza de los actos judiciales no debe circunscribirse sólo a los que emanan de los Jueces de la República. En este marco argumentativo, es entendible y evidente que un Estado pueda atribuir funciones judiciales a órganos diferentes del Poder Judicial para revestirlos de la competencia de proferir verdaderas sentencias judiciales.

Por lo anterior, resulta menester interpretar el Art. 33 del Tratado de Creación del Tribunal de Justicia y los Arts. 122, 123, 127 y 128 del Estatuto, cuando se refieren a los Jueces Nacionales, de manera amplia, en aras de identificar el sujeto legitimado para solicitar la interpretación prejudicial y que dentro de un País Miembro es aquel que ostenta la función judicial. Como la finalidad de la interpretación prejudicial es la aplicación uniforme de la normativa comunitaria por parte de los Jueces Nacionales, los organismos a los cuales el País Miembro ha otorgado funciones judiciales deben acceder a la interpretación prejudicial para cumplir con la filosofía de la misma.

Como conclusión, el término "Juez Nacional" debe interpretarse incluyendo a los organismos que cumplen funciones judiciales, siempre que cumplan las condiciones mínimas señaladas por la ley interna; para de esta manera tenerlos como legitimados para solicitar la interpretación prejudicial, cuando en el ejercicio de dichas funciones conozcan de un proceso en el que deba aplicarse o se controvierta algunas de las normas que integran el Derecho comunitario Andino. Esto consolida el principio de cooperación y colaboración entre el "juez nacional" y el "juez comunitario" en la administración de justicia, ya que ambos con jurisdicción y competencia propias efectúan su aporte a la vigencia del derecho de integración (Proceso 14-IP-2007, pp. 7-8; Proceso 130-IP-2007, p.10).
Cabe indicar que dentro del Proceso 121IP-2014, el TJCA apuntó que cuando se toma en cuenta un criterio funcional, material u objetivo y no únicamente un criterio orgánico, formal o subjetivo, necesariamente se debe ampliar el concepto de juez nacional. Los estados también pueden atribuir funciones jurisdiccionales a órganos diferentes del Poder Judicial, revestidos de nuevas competencias a fin de aplicar normas jurídicas para resolver controversias y emitir decisiones firmes (Proceso 121-IP-2014, p. 7). En particular, "la imparcialidad de estas entidades motiva la 'jurisdiccionalización' de los procedimientos que tramitan, llegando a ser consideradas como 'órganos administrativos sui generis o especiales', u 'órganos de naturaleza híbrida, jurisdiccional-administrativa"' (Proceso 121IP-2014, p. 8).

En el marco de su razonamiento, el TJCA toma en consideración que todos los órganos e instituciones de la administración pública de los Países Miembros deben actuar bajo el principio de legalidad. En ese sentido:

Si las entidades administrativas que ejercen funciones jurisdiccionales cuentan con la interpretación prejudicial del Tribunal antes de la emisión de sus actos administrativos; en la práctica, por una parte se asegura la aplicación uniforme de la norma comunitaria desde el primer momento en que es invocada $y$, por otra, se limita la discrecionalidad de la administración, otorgando mayor seguridad jurídica y predictibilidad a estos procedimientos, aspecto que redunda en una mayor protección de los derechos de los administrados (Proceso 121-IP-2014).

Adicionalmente, el TJCA advirtió que "resulta evidente que no todos los actos administrativos son impugnados en la vía judicial". De tal forma que, si no se habilita el mecanismo de la interpretación prejudicial facultativa para las entidades administrativas que resuelven controversias en los que se aplica el ordenamiento jurídico andino, existe el riesgo de que se consoliden actos que contengan interpretaciones divergentes e inclusive contrarias a la normativa y a la jurisprudencia andinas. EI TJCA concluye señalando que "esta situación podría generar un incumplimiento por parte del País Miembro, ya que los Estados son los principales sujetos del Derecho comunitario y son ellos quienes 
responden por los actos de sus instituciones" (Proceso 121IP-2014, p. 8).

Como la finalidad última de la interpretación prejudicial es la aplicación uniforme de la normativa comunitaria en todos los Países Miembros, los jueces, los árbitros en derecho (Proceso 3-AI-2010), así como las autoridades administrativas a las que dichos Estados han otorgado funciones jurisdiccionales ${ }^{(9)}$ tienen la posibilidad de solicitar la interpretación del TJCA cuando conozcan de un proceso o procedimiento en el que deba aplicarse normativa andina, siempre que la sentencia, el laudo o la resolución sea susceptible de recursos ordinarios en el derecho interno (Proceso 121-IP-2014). En cambio, cuando se trate de la única o última instancia ordinaria, se deberá suspender el proceso y solicitar la correspondiente interpretación prejudicial.

Al efecto, el TJCA estableció algunos criterios generales sobre la naturaleza y funciones de los órganos administrativos consultantes, los mismos que servirán como parámetro de análisis para verificar en cada caso si pueden ser considerados como "juez nacional" (Proceso 121-IP-2014, p. 9).

En consecuencia, el TJCA concluyó en el Proceso 121-IP. 2014 que:

En la primera oportunidad en la que un órgano o entidad administrativa realice una consulta con el propósito de obtener la interpretación prejudicial, esta deberá acreditar que: 1) se ha constituido por mandato legal, 2) se trata de un órgano permanente, 3) el carácter obligatorio de sus competencias, 4) el deber de aplicar normas comunitarias andinas en el ejercicio de sus competencias, 5) el carácter contradictorio de los procedimientos a su cargo y el respeto al debido proceso; y, 6) la imparcialidad de sus actos". En el caso particular, el TJCA verificó que "la Dirección de Signos Distintivos y la Comisión de Signos Distintivos son órganos dentro de la estructura orgánica del Instituto Nacional de Defensa de la Competencia y de la Propiedad Intelectual (INDECOPI), de la República del Perú, constituidas por mandato legal con carácter permanente, cuya jurisdicción es obligatoria, asumiendo competencia primaria exclusiva para conocer y resolver el procedimiento aplicando la normativa andina. Asimismo, el presente procedimiento respeta el debido proceso y posee el carácter contradictorio, además de que debido a su autonomía funcional queda garantizada su imparcialidad (Proceso 121-IP2014, p. 9)

Como podemos observar, el Proceso 121-IP-2014 guarda una íntima relación con los principios del Derecho administrativo, a saber: el principio de legalidad (las autoridades administrativas deben actuar con respeto al Derecho comunitario dentro de las facultades que le estén atribuidas), el debido procedimiento, el principio de impulso de oficio (las autoridades deben dirigir e impulsar de oficio el procedimiento y ordenar la realización o práctica de los actos que resulten convenientes para el esclarecimiento y resolución de las cuestiones necesarias), el principio de imparcialidad (las autoridades administrativas actúan sin ninguna clase de discriminación entre los administrados, resolviendo conforme al ordenamiento jurídico comunitario) y el principio de verdad material (en el procedimiento la autoridad administrativa deberá verificar plenamente los hechos que sirven de motivo a sus decisiones, para lo cual deberá adoptar todas las medidas necesarias).

Cuando un consultante solicita interpretación prejudicial surgen dos obligaciones jurídicas: (i) aplicar la interpretación al momento de resolver el caso; y, (ii) enviar la sentencia, el laudo o la resolución administrativa al TJCA para que se verifique la debida aplicación de la interpretación prejudicial (c.f. García, 2014, pp. 142-162). Pero, como apuntamos anteriormente, en la mayoría de los casos no se cumple con esta obligación de fiscalización y control por parte de los Países Miembros y la SGCA.

Tras analizar la evolución jurisprudencial del TJCA en relación con la ampliación de la legitimidad activa para solicitar interpretaciones prejudiciales, creemos positivas las decisiones que incluyen a los árbitros y a los órganos administrativos encargados del registro de la Propiedad Industrial. Ello guarda concordancia con la propia autonomía del Derecho comunitario andino y el objeto y fin último del mecanismo de la interpretación prejudicial, cual es precisamente, la interpretación y la aplicación uniforme del Derecho comunitario en el territorio de los cuatro Países Miembros.

Una interpretación amplia del concepto de "juez nacional" asegura la plena identificación del legítimo operador nacional, considerado también como un "juez comunitario" por colaborar en la interpretación y la aplicación uniforme del Derecho comunitario (Mendoza, 2019, p. 271). En suma, se ha privilegiado la interpretación teleológica o funcional, ya que esta se adapta mejor para lograr la

(9) Se incluye a las entidades administrativas que tramitan procedimientos "jurisdiccionalizados". 
uniformidad en la interpretación y la aplicación del Derecho comunitario en todos los países andinos.

\section{Un breve paralelismo entre la consulta prejudicial del Tribunal de Justicla de la UE (TJUE) y la del TJCA}

Al ser la CAN y la UE procesos de integración supranacionales, el TJCA sigue muy de cerca el desarrollo de la jurisprudencia europea y ha recogido de manera uniforme los tres principios del efecto directo, de la aplicación inmediata y de la primacía del Derecho comunitario, consagrados en Van Gend \& Loos, Flaminio Costa y Simmenthal (Asunto 26/02, Van Gend \& Loos, 5 de febrero de 1963; asunto 6/64, Flaminio Costa vs ENEL, 15 de julio de 1963; asunto 106/77, Simmenthal, 9 de marzo de 1978), que fundamentan la esencia de la integración europea y que son el fruto de las consultas prejudiciales realizadas por las distintas cortes nacionales. Adicionalmente, en el ámbito de la PI, el TJCA ha citado reiteradamente la doctrina del TJUE en sus fallos (Proceso 13-IP-2004, Proceso 179-IP-2006, Proceso 31-IP-2010; Vigil, 2011, p. 2).

Asimismo, el TJCA tampoco ha dejado de emplear e invocar la doctrina europea. Ricardo Alonso García, Robert Lecourt, Santiago Muñoz Machado, Gil Carlos Rodríguez Iglesias o Dámaso Ruiz-Jarabo Colomer son algunos de los autores mencionados (c.f. Suárez, 2001, p. 94).

Sin embargo, en el ámbito de la legitimidad activa para solicitar consultas prejudiciales ante el TJUE podemos advertir una clara diferencia. Esta legitimidad activa es mucho más restringida, toda vez que las entidades puramente administrativas (p.e. los órganos administrativos encargados del registro de la Propiedad Industrial) y los árbitros no pueden formular consultas. En efecto, el Tribunal sostuvo que no tenía jurisdicción para responder la consulta remitida ya que "el tribunal remitente actuó dentro de sus facultades administrativas y no judiciales" (TJUE, asunto C-96/04, 27 de abril del 2006). En el caso de los árbitros (cuya composición dependa de las partes en conflicto), el TJUE ha denegado la calificación de "órgano jurisdiccional", pues la falta de pertenencia de los árbitros a un sistema jurisdiccional estatal impide que estos puedan remitir cuestiones prejudiciales (TJCE, asunto 102/81, 23 de marzo de 1982).

No obstante, el Tribunal de Luxemburgo admitió varias cuestiones prejudiciales solicitadas por órganos que formalmente no son jurisdiccionales como, por ejemplo, los colegios profesionales en el ejercicio de sus funciones disciplinarias o los tribunales arbitrales de carácter permanente. En el caso español, se han admitido cuestiones planteadas por el Tribunal Central Económico Administrativo, el Tribunal de Defensa de la Competencia o el Tribunal Catalán de Contratos del Sector Público sobre la base de los criterios esgrimidos por el propio Tribunal: el origen legal del órgano, su permanencia, el carácter contradictorio del procedimiento, la aplicación por parte del órgano de normas jurídicas (TJUE, asunto C-203/01, 06 de noviembre del 2015), así como su independencia e imparcialidad.

En segundo lugar, otra diferencia con la consulta prejudicial de la UE consiste en que a nivel de la CAN no operan las teorías del "acto claro" y del "acto aclarado". En el seno de la UE ellas juegan como excepciones a la obligación de planteamiento de la cuestión prejudicial de interpretación (Bueno y Perotti, 2005). Para el TJCA, la claridad de una disposición o "[l]a existencia de un pronunciamiento anterior del Tribunal, así se refiera a la misma materia debatida en un proceso ulterior, no exime al juez nacional de esta última causa de su obligación de elevar la correspondiente solicitud de interpretación" (Proceso 3-IP-89, p. 3). Ello es así debido al efecto relativo e inter partes de la interpretación prejudicial, que solo vincula a los jueces involucrados en el caso concreto y no a los jueces del bloque regional (cf. Klein, 2011, p. 75). En palabras del TJCA: "la interpretación que realiza el Tribunal es para cada caso concreto" (Proceso 1-IP-87, p. 4).

Según Tobón, "[e]l fundamento de dicha posición es que el TJCA podría variar y cambiar su opinión cuando encuentre razones justificadas para hacerlo. El juez nacional debe solicitar la interpretación prejudicial siempre, aun en los casos similares o análogos, ya que en el ámbito andino no puede hablarse de una 'precedencia' interpretativa" (Tobón, 2005, pp. 461 y ss., p. 469).

Cabe indicar que un gran sector de la doctrina opina que el TJCA debería revisar su posición sobre acoger, al menos, la teoría del acto aclarado, aunque bajo ciertas premisas, exigencias y circunstancias jurídicas, tendentes a evitar los abusos. Siguiendo, entre otros, a Bueno \& Perotti (2005), se es de la opinión que la actual existencia de una amplia jurisprudencia sobre PI (que abarca cuestiones como los requisitos que debe reunir un signo para ser registrable, las marcas denominativas, gráficas o mixtas, la semejanza entre signos o los signos evocativos) permitiría al juez nacional abstenerse de efectuar la consulta 
sobre dichas nociones o los preceptos en los que se emplean, dando directamente aplicación a dicha jurisprudencia. De esta forma, se evitaría la acumulación ante el TJCA de asuntos sobre la misma materia, que dan lugar a sentencias muy semejantes, o incluso idénticas, que desvalorizan las funciones del Tribunal (Vigil, 2004, p. 145; Troya, 2014).

En tercer lugar, en ese paralelismo entre el TJCA y el TJUE, algunos autores han llamado la atención sobre el hecho que, en el ámbito andino y mayormente antes del 2014, ha faltado el apoyo de un jurist advocacy movement, que sí concurrió en el continente europeo. Este concepto engloba al conjunto de abogados, jueces, académicos, asesores y funcionarios nacionales y europeos que se organizaron, desde el inicio de la integración europea, para defender los objetivos del proceso de integración regional (Alter, 2016, p. 4).

Para concluir este breve análisis, creemos oportuno recoger las palabras de Alonso García:

Esta inspiración en el sistema europeo, por lo demás, no se traduce en una importación irreflexiva de principios y técnicas jurídicas, manteniendo el Tribunal como propios del sistema andino rasgos ajenos a los configurados por el Tribunal europeo de Justicia (...) no solo la experiencia europea puede y debe servir para consolidar e intensificar los sistemas de integración en Latinoamérica. Antes al contrario, también la Unión Europea debería prestar atención al desarrollo de estos, a los efectos de tener en cuenta posibles elementos con los que perfeccionar y enriquecer un modelo que hace gala, desde sus orígenes, de un dinamismo en constante evolución (Alonso, 2008, pp. 73 y 84).

\section{Algunos apuntes sobre cómo se deben enfrentar los cambios jurisprudenciales del TJCA}

En el seno del TJCA se han gestado diversas modificaciones jurisprudenciales que han iniciado una nueva línea de pronunciamientos. Ante ello surgen varias interrogantes, como el qué hacer ante dichos cambios jurisprudenciales. En otras palabras, cuál es el destino de los asuntos resueltos sobre la base de la jurisprudencia anterior.

A nuestro parecer, como regla general, cada interpretación prejudicial se emite individualmente para cada caso concreto y debe primar la seguridad jurídica sobre lo resuelto. En ese sentido, en principio, no cabe jurisprudencia que se pudiera aplicar retroactivamente. De esta forma, la nueva jurisprudencia se debe aplicar a los procedimientos o procesos en curso. Sin embargo, se pueden esbozar ciertas posibles excepciones a la regla general cuando se distingue entre los procedimientos o procesos en curso y los finalizados. Si bien las interpretaciones prejudiciales son aplicables para el caso concreto, no se puede negar que, en el fondo, se trata de decisiones judiciales que tienen ciertas implicaciones normativas que van más allá del contexto del caso particular en el que se pronunció.
Por un lado, en el caso de los procedimientos o procesos en curso, la autoridad que conozca dicho proceso deberá adoptar prima facie las interpretaciones prejudiciales previas que pudieran existir en base a dicha interpretación del Tribunal y que hemos identificado como la regla general: "El juez que conozca del proceso interno en que se formuló la consulta, deberá adoptar en su sentencia la interpretación del Tribunal" (ETJCA, art. 127).

Sin embargo, si bien la interpretación prejudicial emitida por el TJCA resulta aplicable a lo largo del proceso, ya sea en sede administrativa o judicial; podría solicitarse de manera excepcional una nueva consulta cuando: la solicitud de interpretación prejudicial verse sobre diferentes normas del ordenamiento jurídico comunitario; la existencia de pruebas o documentos que determinan la aparición de nuevos elementos de juicio, no tomados en cuenta en el momento de realizar la interpretación prejudicial original; si a criterio del consultante existen temas o asuntos sobre los que no se pronunció el TJCA; o, si a criterio del consultante es necesaria la ampliación o la aclaración de la interpretación prejudicial (Proceso 121-IP-2014).

Por lo tanto, en el caso de los procedimientos o procesos en curso, se podría intentar solicitar una nueva interpretación prejudicial debido a que se ha producido un cambio jurisprudencial en el seno del TJCA (la aparición de nuevos elementos de juicio), a fin de promover en el caso concreto un segundo pronunciamiento del TJCA. Ello no impide que las autoridades apliquen por propia iniciativa la nueva línea jurisprudencial. Tal fue el caso de la Resolución 656-2019/TPI-INDECOPI, mediante la cual la Sala Especializada en Propiedad Intelectual del Tribunal del INDECOPI revocó la Resolución 5204-2018/CSD-INDECOPI (basada en la jurisprudencia anterior del Proceso 99-IP2014), en aplicación de la jurisprudencia actual del TJCA. En específico, se declaró infundada la acción de nulidad interpuesta por Construcciones Inmobiliarias FP S.A.C. contra el registro de la marca $Q A P A Q$, toda vez que resultó insuficiente el ámbito de influencia del nombre comercial CAPAC. 
Por otro lado, en los procedimientos o procesos finalizados, al resultar materialmente imposible solicitar una nueva interpretación prejudicial, en caso lo permitiera la legislación nacional, cabe preguntarse si se podría intentar la nulidad de la resolución administrativa, el laudo arbitral (Proceso 3-Al2010) o la sentencia por contravenir el Derecho comunitario andino, es decir, la jurisprudencia actual. Esto sólo podría imaginarse siempre y cuando la respectiva legislación nacional lo permitiera; por ejemplo, si en sede nacional existiera un recurso extraordinario de revisión, basado en un hecho nuevo que pudiera influir de manera decisiva en el resultado del mismo y que hubiera sido desconocido en la fecha de la emisión de la sentencia. De esta manera, dentro de dicho proceso de nulidad o de revisión se podría solicitar una interpretación al TJCA; no sobre el artículo específico de la Decisión 486, cuya interpretación ha cambiado. Esta interpretación del TJCA sería sobre el objeto y el alcance de una interpretación prejudicial y su relación con los cambios jurisprudenciales, debido a que una interpretación posterior varió el alcance y el ámbito de aplicación de una norma jurídica, modificando así el propio objeto de los derechos del titular.

A manera ilustrativa, en el caso peruano, si un juez aplica la jurisprudencia anterior en desmedro de la que rige actualmente y en contra una sentencia que haya adquirido calidad de cosa juzgada, cabría presentar una demanda de nulidad de cosa juzgada fraudulenta por haberse violado el debido proceso. De manera alternativa, podría intentarse una acción de amparo ante el Tribunal Constitucional, alegando que se trata de una sentencia derivada de un proceso irregular que vulnera el debido proceso. En ambos casos el juez nacional que resuelva como última instancia ordinaria también se encontraría obligado a solicitar una interpretación prejudicial (c.f. Gálvez, 2001, pp. 142-143).

Tras proyectar preliminarmente algunas posibles excepciones a la regla general cuando distinguimos entre los procedimientos o procesos en curso y los finalizados, consideramos que, al encontrarse la jurisprudencia andina en constante evolución, resulta necesario -para cualquier ordenamiento jurídico- encontrar un sano equilibrio entre la estabilidad y la seguridad jurídica, unida a la flexibilidad para hacer posible la evolución jurisprudencial.

Por ejemplo, dentro del Proceso 3-AI-2010, se aprecia que el TJCA estableció, por primera vez, la obligación de los árbitros en derecho de solicitar interpretación prejudicial al TJCA cuando los procesos arbitrales versen sobre Derecho comunitario. En el caso citado, en aras de un sano equilibrio entre la estabilidad, la seguridad jurídica y la flexibilidad que haga posible la evolución jurisprudencial; cabe preguntarse si se puede permitir que esta nueva obligación (desconocida por los propios árbitros) se aplique retroactivamente, provocando la nulidad de laudos arbitrales por no haberse cumplido con dicha obligación. En otras palabras, cabría pretender que dicha nueva obligación de solicitar interpretación prejudicial se aplique con carácter retroactivo a laudos que se emitieron cuando esa "norma jurisprudencial" no se podía conocer porque sencillamente no existía ${ }^{(10)}$.

A nuestro parecer, en dicho proceso se pudo matizar y limitar las consecuencias de los cambios jurisprudenciales del TJCA. En otras palabras, el juez comunitario tiene la facultad de limitar o atemperar los efectos retroactivos que se adjudican a su decisión cuando lo hace necesario el principio de seguridad jurídica (ETJCA, art. 106). Sin embargo, para ello, hay que estar siempre al caso concreto a efectos de ponderar aspectos fundamentales como la seguridad jurídica y la garantía de los derechos.

Sobre el alcance temporal de las consecuencias que se puedan derivar de un cambio jurisprudencial, el TJCE "por consideraciones imperiosas de seguridad jurídica" y a "título excepcional" permitió que la solución nueva sea aplicada sólo a los recursos presentados después de la fecha de la sentencia y a las situaciones y actos jurídicos posteriores (TJCE, asunto 42/59 y 49/59, SNUPAT/Alta Autoridad, 22 de marzo de 1961).

Para concluir, consideramos relevante traer a colación lo señalado por Blasco Gascó (2009):

El cambio de criterio jurisprudencial, y en concreto, su eficacia temporal retroactiva debe ser al menos templada en los siguientes supuestos:

1. Cuando el cambio de criterio introduzca un obstáculo o un requisito procesal o sustantivo no exigido por la doctrina jurisprudencial en el momento de presentar la demanda o de producirse la situación (relación) jurídico material.

2. Cuando el cambio de criterio se refiera a relaciones jurídicas duraderas o de trato

(10) Véase Zejalbo, J. (2019). Notas sobre la retroactividad de la jurisprudencia. https://www.notariosyregistradores.com/doctrina/ ARTICULOS/2012-retroactividad.htm. 
sucesivo. Para el autor la nueva doctrina jurisprudencial no se puede extender a las prestaciones y situaciones que sean debidas o nazcan después del cambio de criterio.

3. Cuando el cambio de criterio suponga una situación objetivamente menos beneficiosa para el sujeto en el sentido de limitar o restringir un derecho o facultad que, de acuerdo con el criterio anterior, había sido declarado válido y que el nuevo criterio, sin mediar modificación legislativa, declara nulo.

Cuando el cambio de criterio obedezca a una interpretación de la norma nacional con base a una directiva comunitaria aún no transpuesta y en periodo de serlo.

Finalmente, no parece que haya inconveniente en que el Tribunal anuncie un cambio de criterio jurisprudencial para el futuro en un pronunciamiento que necesariamente será obiter (pp. 854 - 855).

Sea como fuere, confiamos en que la presente contribución sirva para iniciar el debate y permita promover próximos estudios sobre los efectos y las consecuencias jurídicas de los cambios jurisprudenciales del TJCA.

\section{Conclusiones}

Las interpretaciones prejudiciales señalan el derrotero y expresan los avances positivos hacia la consolidación del proceso de integración jurídica andino, a diferencia de las acciones de incumplimiento y de nulidad, surgidas como consecuencia de las fallas o imponderables del proceso de integración. En ese sentido, en la CAN se ha llegado a consolidar un verdadero Estado de Derecho en la protección de la Propiedad Intelectual gracias a la jurisprudencia del Tribunal.

Entre las mayores ventajas de la unificación jurisprudencial o del establecimiento de criterios de obligatorio cumplimiento por parte del TJCA en materias específicas de la Propiedad Intelectual (entre otras) están las siguientes: (i) la racionalización de la demanda por interpretaciones prejudiciales facultativas (menos necesidad de acudir siempre al Tribunal comunitario); y (ii) un mayor conocimiento y aplicación de Decisiones andinas y de su interpretación jurisprudencial en las controversias que surjan en los países, en beneficio del Derecho comunitario.

Si bien el TJCA sigue de cerca la jurisprudencia europea, en el ámbito de la legitimidad activa para solicitar consultas prejudiciales ante el TJUE podemos advertir una clara diferencia. Esta legitimidad activa es mucho más restringida, toda vez que las entidades puramente administrativas (p.e. los órganos administrativos encargados del registro de la Propiedad Industrial) y los árbitros no pueden formular consultas. No obstante, el Tribunal de Luxemburgo admitió varias cuestiones prejudiciales solicitadas por órganos que formalmente no son jurisdiccionales como, por ejemplo, los colegios profesionales en el ejercicio de sus funciones disciplinarias o los tribunales arbitrales de carácter permanente.

El Tribunal Andino, sobre la base de un criterio funcional, material u objetivo, ha interpretado de manera amplia y generosa el concepto de "juez nacional"; incluyendo a los órganos administrativos de los Países Miembros, así como a los árbitros. En particular, con la finalidad de verificar la legitimidad activa de los órganos administrativos encargados del registro de la Propiedad Industrial para solicitar interpretación prejudicial facultativa, el TJCA tomó en consideración: el principio de autonomía del Derecho comunitario andino; el objeto y la finalidad de la interpretación prejudicial; la naturaleza de los actos emitidos por las autoridades nacionales; el fenómeno de la jurisdiccionalización de los procedimientos tramitados en sede administrativa; y, el principio de legalidad. De tal manera que, mediante las interpretaciones prejudiciales emitidas en los procesos 105-IP-2014, 121 IP-2014, 242-IP-2015 y 737-IP-2018, el TJCA reconoció la legitimidad activa de los cuatro órganos administrativos encargados del registro de la Propiedad Industrial de la CAN.

Las autoridades administrativas, los árbitros y los jueces nacionales se encuentran en la obligación de no contravenir la normativa y la jurisprudencia andinas al momento de emitir sus resoluciones administrativas, laudos o sentencias, respectivamente. De lo contrario, el particular afectado podría activar la acción de incumplimiento en contra del País Miembro o incluso la acción de indemnización por daños y perjuicios por incumplimiento del Derecho comunitario.

Como regla general, la interpretación prejudicial se emite para cada caso concreto y debe primar la seguridad jurídica sobre lo resuelto. En ese sentido, en principio, no cabría una jurisprudencia que se pudiera aplicar retroactivamente. De esta forma, la jurisprudencia nueva, se tendría que aplicar a los procedimientos o procesos en curso.

Sin embargo, se pueden esbozar ciertas posibles excepciones a la regla general cuando distinguimos entre los procedimientos 
o procesos en curso y los finalizados. Si bien las interpretaciones prejudiciales son aplicables para el caso concreto, no podemos negar que, en el fondo, se trata de decisiones judiciales que tienen ciertas implicaciones normativas que van más allá del contexto del caso particular en el que se pronunció.

\section{Referencias bibliográficas}

Alonso, R. (2008). Un paseo por la jurisprudencia supranacional europea y su reflejo en los sistemas suramericanos de integración. Advocatus Ediciones.

Alter, K. (2006). Private Litigants and the New International Courts. Comparative Political Studies, 39(1), 22-49.

Alter, K. (2016). Jurist Advocacy Movements in Europe and the Andes. iCourts Working Paper Series, 65. https://doi. org/10.1177/0010414005283216

Alter, K. \& Helfer, L. (2017). Transplanting International Courts: The Law and Politics of the Andean Tribunal of Justice (International Courts and Tribunals Series). Oxford University Press. http://dx.doi. org/10.2139/ssrn.1270075

Blasco Gascó, F. (2009). Eficacia retroactiva y prospectiva del cambio de criterio jurisprudencial. Comentario de la Sentencia del Tribunal Supremo del 11 de septiembre de 2009. En M. Ysquierdo Tolsada (coord.). Comentarios a las sentencias de unificación de doctrina: civil y mercantil, 3 (pp. 829- 880). Dykinson.

Bueno, P. \& Perotti, A. (2005). La teoría del acto aclarado ¿resulta necesaria su aplicación en el marco de la interpretación prejudicial andina? Dikaion, 14(19), 133-152.

Cienfuegos, M. (2013a). Cuestiones prejudiciales en la Unión Europea y consultas prejudiciales en la Comunidad Andina: similitudes, diferencias e influencias. Revista Electrónica de Estudios Internacionales, 25, 1-43.

Cienfuegos, M. (2013b). Consultas prejudiciales en la Comunidad Andina y cuestiones prejudiciales en la Unión Europea: ensayo de comparación, Revista de la Facultad, 4(1), Nueva Serie II, 37-77.

Comunidad Andina de Naciones. (1996). Protocolo Modificatorio del Tratado de Creación del Tribunal de Justicia de la Comunidad Andina. http:// http://idatd.cepal.org/Normativas/CAN/Espanol/Tratado_de_ Creacion_del_Tribunal_de_Justicia.pdf.

Corte Constitucional de Colombia. (1995, 25 de mayo). Sentencia No. C-228/95 recaída en Expediente D-576. http://www.corteconstitucional. gov.co/ relatoría/1995/C-228-95.htm.

Diez Canseco, L. (2019). Patience and Perseverance in Administering Justice - The Role of a Judge at the Andean Tribunal of Justice. En Selvik, G., Clifton, M. J., Haas, T., Lourenco, L. \& Schwiesow, K. (eds.), The Art of Judicial Reasoning (pp. 191-200). Springer.

Gálvez, M. (2001). Comentarios sobre la interpretación prejudicial del Tribunal de Justicia de la Comunidad Andina, Themis Revista de Derecho, 42, 31-144.

García, G. (2014). Análisis de la Interpretación Prejudicial emitida por el Tribunal de Justicia de la Comunidad Andina dentro del Proceso 242IP-2015 (Marca: TEXTURA SUPERFICIE "OLD PARR"). Foro: Revista de Derecho, 22, 143-162.c http://dx.doi.org/10.2139/ssrn.1270075
García de Enterría, E. \& Fernández, T. (2011) Curso de Derecho Administrativo (Tomo II). Thomson Reuters.

Helfer, L., Alter, K. \& Guerzovich, M. (2009). Islands of effective international adjudication: Constructing an intellectual property rule of law in the Andean Community. The American Journal of International Law, 103(1), 1-47. https://doi:10.2307/20456720.

Ibañez, M. (2010). La aplicación del Derecho fiscal europeo y el control de la adaptación del ordenamiento interno al comunitario: la cuestión prejudicial y legitimación de los TEA. Cuadernos de Formación, 11, 179-209.

Klein, L. (2011). Interpretación y aplicación uniforme del Derecho de la Integración: Unión Europea, Comunidad Andina y MERCOSUR. BdeF.

Mendoza, Y. (2019). La interpretación sobre el juez nacional en el Tribunal de Justicia de la Comunidad Andina. Agenda Internacional, 26(37), 271-292. https://doi.org/10.18800/agenda.201901.010

Santos, J. (2013). El Derecho Administrativo del sistema comunitario Andino. Revista Digital de Derecho Administrativo, 10, 21-44.

Sasaki, M. (2014). El régimen común sobre propiedad industrial en la Comunidad Andina tras la adopción de la Decisión 689: ¿Integración regional o acuerdos bilaterales? En Téllez, E. \& Oliveira, R. (dirs.), Derecho Internacional en la Práctica Latinoamericana (pp. 67-85). Tirant lo Blanch.

Suárez, J. (2001). Integración y Supranacionalidad en la Comunidad Andina: Proceso decisorio, Sistema jurisdiccional y relación con los Derechos nacionales [Tesis para optar por el título de Doctor, Universidad Complutense de Madrid]. Repositorio UCM. https://eprints.ucm.es/id/eprint/7299/.

Suárez, J. (2006). La Responsabilidad Patrimonial del Estado y el Derecho Comunitario: una visión europea, andina y venezolana. Editorial Sherwood.

Tobón, N. (2005). La doctrina del acto claro y la interpretación prejudicial en la Comunidad Andina, Vniversitas, 54(109), 461-482.

Tribunal de Justicia de la Comunidad Andina (1987). Proceso 1-IP-87.

Tribunal de Justicia de la Comunidad Andina. (1989). Proceso 3-IP-89.

Tribunal de Justicia de la Comunidad Andina. (1996). Protocolo Modificatorio del Tratado de Creación del Tribunal de Justicia de la Comunidad Andina.

Tribunal de Justicia de la Comunidad Andina (2001). Estatuto del Tribunal de Justicia de la Comunidad Andina. https://www.tribunalandino. org.ec/sitetjca1/TEstatuto\%20del\%20Tribunal\%20 
de\%20Justicia\%20de\%20la\%20Comunidad\%20 Andina.pdf.

Tribunal de Justicia de la Comunidad Andina. (2004). Proceso 13-IP-2004.

Tribunal de Justicia de la Comunidad Andina. (2006). Proceso 179-IP-2006.

Tribunal de Justicia de la Comunidad Andina. (2010a). Proceso 31-IP-2010.

Tribunal de Justicia de la Comunidad Andina. (2010b). Proceso 3-Al-2010.

Tribunal de Justicia de la Comunidad Andina. (2013). Proceso 181-IP-2013.

Tribunal de Justicia de la Comunidad Andina. (2014a). Proceso 248-IP-2013.

Tribunal de Justicia de la Comunidad Andina. (2014b). Proceso 105-IP-2014.

Tribunal de Justicia de la Comunidad Andina. (2014c). Proceso 121-IP-2014.

Tribunal de Justicia de la Comunidad Andina. (2015a). Proceso 297-IP-2015.

Tribunal de Justicia de la Comunidad Andina. (2015b). Proceso 242-IP-2015.

Tribunal de Justicia de la Comunidad Andina. (2016). Informe de Labores de Gestión 2015. https://www.tribunalandino.org.ec/wp-content/ uploads/2019/04/INFORME2015.pdf

Tribunal de Justicia de la Comunidad Andina. (2018). Informe de Labores de Gestión 2017. https://www.tribunalandino.org.ec/wp-content/ uploads/2019/ 04/ INFORME2017.pdf.

Tribunal de Justicia de la Comunidad Andina. (2020). Informe de Labores de Gestión 2019. https://www.tribunalandino.org.ec/transparencia/ gestion /2019/INFORMES2019.pdf.

Tribunal de Justicia de la Comunidad Europea.(1961, 22 de marzo). Sentencia del Tribunal de Justicia de 22 de marzo de 1961. Asuntos acumulados $42 / 59$ y $49 / 59$, SNUPAT vs Alta Autoridad. https://eurlex.europa.eu/legalcontent/ES/TXT/ $\mathrm{PDF} /$ ?uri=CELEX:61959CJ0042\&from=ES.
Tribunal de Justicia de la Comunidad Europea. (1963, 5 de mayo). Sentencia del Tribunal de Justicia de 5 de febrero de 1963. Asunto $26-$ 62, Van Gend \& Loos. https://eur-lex.europa.eu/legal-content/ES/TXT/ PDF/?uri=CELEX:61962CJ0026\&qid $=1614460859348 \&$ from $=E S$

Tribunal de Justicia de la Comunidad Europea. (1964, 15 de julio). Sentencia del Tribunal de Justicia de 15 de julio de 1964. Asunto 6/64 Flaminio Costa vs ENEL. https://eur-lex.europa.eu/legal-content/ES/ TXT/PDF/?uri= CELEX:61964CJ0006\&from=ES

Tribunal de Justicia de la Comunidad Europea. (1978, 9 de marzo). Sentencia del Tribunal de Justicia de 9 de marzo de 1978. Asunto 106/77, Simmenthal. https://eur-lex.europa.eu/legal-content/ES/TXT/ PDF/?uri=CELEX:61977CJ0106\&from=ES

Tribunal de Justicia de la Comunidad Europea. (1982, 23 de marzo). Sentencia del Tribunal de Justicia de 23 de marzo de 1982. Asunto 102/81, Nordsee. http://curia.europa.eu/juris/showPdf.jsf?text=\&docid $=91402 \&$ pagelndex $=0 \&$ doclang $=E S \&$ mode $=\mid s t \& d i r=\& o c c=$ first $\partial=1 \&$ cid=1104761.

Tribunal de Justicia de la Unión Europea. (2006, 27 de abril). Sentencia del Tribunal de Justicia (Sala Primera) de 27 de abril de 2006, Asunto C-96/04. http://curia.europa.eu/juris/showPdf. jsf?text $=$ \&docid $=56256$ \&pagelndex $=0$ \&doclang $=E S \&$ mode $=\mid s t \& d i r=$ \&occ $=$ first \&part $=1 \&$ cid $=6676912$.

Tribunal de Justicia de la Unión Europea. (2015, 6 de octubre). Sentencia del Tribunal de Justicia (Gran Sala) de 6 de octubre de 2015 , Asunto C-203/14. http://curia.europa.eu/juris/document/document. jsf?text $=$ \&docid $=169181$ \&pagelndex $=0$ \&doclang $=E S \&$ mode $=I$ st\&dir $=$ \&occ $=$ first \&part $=1 \&$ cid $=6677975$.

Troya, J. (2014). Comentarios y sugerencias del TJCA al Proyecto de Protocolo modificatorio del TCTJCA [Memorándum].

Vigil, R. (2004). La cooperación entre los órganos jurisdiccionales nacionales y el Tribunal de Justicia de la Comunidad Andina: la consulta prejudicial. Testimonio Comunitario - Doctrina, Legislación, Jurisprudencia (pp. 96-109). Tribunal de Justicia de la Comunidad Andina. http://www.tribunalandino.org.ec/libros/testimonio_ comunitario.pdf.

Vigil, R. (2012). El reflejo de la jurisprudencia europea en los fallos del Tribunal de Justicia de la Comunidad Andina: aspectos teóricos y pragmáticos. En Hernández, M \& Deluca, S. (Coords.) Tribunales en organizaciones de integración. Mercosur, Comunidad Andina y Unión Europea (pp. 215-236) Aranzadi Thomson Reuters.

Zejalbo, J. (2019). Notas sobre la retroactividad de la jurisprudencia. https://www.notariosyregistradores.com/doctrina/ARTICULOS/2012retroactividad.htm. 